\title{
Dynamics of Third-Order Nonlinear Neutral Equations
}

\author{
Hua Wang, ${ }^{1}$ Li Liu, ${ }^{1}$ and Yanxiang Tan ${ }^{2}$ \\ ${ }^{1}$ School of Mathematics and Computing Science, Changsha University of Science and Technology, Changsha, Hunan 410114, China \\ ${ }^{2}$ College of Mathematics and Econometrics, Hunan University, Changsha, Hunan 410082, China
}

Correspondence should be addressed to Li Liu; babaley@163.com

Received 12 March 2014; Accepted 18 June 2014; Published 14 July 2014

Academic Editor: Taishan Yi

Copyright (C) 2014 Hua Wang et al. This is an open access article distributed under the Creative Commons Attribution License, which permits unrestricted use, distribution, and reproduction in any medium, provided the original work is properly cited.

The aim of this paper is to study oscillatory and asymptotic properties of the third-order nonlinear neutral equation with continuously distributed delays of the form $\left(r(t)\left(\left[x(t)+\int_{a}^{b} p(t, \mu) x[\tau(t, \mu)] d \mu\right]^{\prime \prime}\right)^{\alpha}\right)^{\prime}+\int_{c}^{d} q(t, \xi) f^{\alpha}(x[g(t, \xi)]) d \xi=0$. Applying suitable generalized Riccati transformation and integral averaging technique, we present new criteria for oscillation or certain asymptotic behavior of nonoscillatory solutions of this equation. Obtained results essentially improve and complement earlier ones.

\section{Introduction}

In recent years, the dynamics theory such as oscillation theory and asymptotic behavior of differential equations and their applications have been and still are receiving intensive attention [1-4]. In fact, in the last few years several monographs and hundreds of research papers have been written; see, for example, the monograph [5]. Determining oscillation criteria for particular second-order differential equations has received a great deal of attention in the last few years [6-8]. For example, [9] considered

$$
\begin{aligned}
& \left(r(t)[x(t)+p(t) x(t-\tau)]^{\prime}\right)^{\prime} \\
& \quad+\int_{a}^{b} q(t, \xi) x[g(t, \xi)] d \sigma(\xi)=0
\end{aligned}
$$

and obtained oscillatory criteria of Philos type. In [10], by means of Riccati transformation technique, Han et al. established some new oscillation criteria for the second-order Emden Fowler delay dynamic equations on a time scale $T$ :

$$
x^{\prime \prime}(t)+p(t) x^{\gamma}(\tau(t))=0 .
$$

However, compared to second-order differential equations, the study of oscillation and asymptotic behavior of thirdorder differential equations has received considerably less attention in the literature [11-15]. In [16], Qiu investigated the oscillation criteria for the third-order neutral differential equations taking the following form:

$$
\begin{aligned}
(r(t) & {\left.[x(t)+p(t) x(t)]^{\prime \prime}\right)^{\prime} } \\
& +\int_{a}^{b} q(t, \xi) x[g(t, \xi)] d \sigma(\xi)=0, \quad t \geq t_{0} .
\end{aligned}
$$

By using a generalized Riccati transformation and integral averaging technique, Zhang et al. [17] established some new sufficient conditions which ensure that every solution of the following equation oscillates or converges to zero:

$$
\begin{gathered}
\left(r(t)\left(\left[x(t)+\int_{a}^{b} p(t, \mu) x[\tau(t, \mu)] d \mu\right]^{\prime \prime}\right)\right)^{\prime} \\
+\int_{c}^{d} q(t, \xi) f(x[g(t, \xi)]) d \xi=0 .
\end{gathered}
$$

As we know, the dynamics theory such as oscillation theory and asymptotic behavior of the following equation have not been investigated up to now:

$$
\begin{gathered}
\left(r(t)\left(\left[x(t)+\int_{a}^{b} p(t, \mu) x[\tau(t, \mu)] d \mu\right]^{\prime \prime}\right)^{\alpha}\right)^{\prime} \\
+\int_{c}^{d} q(t, \xi) f^{\alpha}(x[g(t, \xi)]) d \xi=0 .
\end{gathered}
$$


With the help of a generalized Riccati transformation and integral averaging technique, this paper aims to establish some new sufficient conditions of Philos type which ensure that every solution of (5) oscillates or converges to zero. Our results improve and complement the corresponding results in $[6,11-17]$. We should point out that, in this paper, $\alpha$ is any quotient of odd positive integers and $\alpha \leq 1$; it is more general than that reported in [17] where $\alpha=1$.

We are interested in (5) in the case of $t \geq t_{0}$. Throughout this paper, we assume that the following hypotheses hold:

$\left(H_{1}\right) r(t) \in C^{1}\left(\left[t_{0}, \infty\right),(0, \infty)\right), \int_{t_{0}}^{\infty}(1 / r(t)) d t=\infty ;$

$\left(H_{2}\right) P(t, \mu) \in C\left(\left[t_{0}, \infty\right) \times[a, b], R\right), 0 \leq p(t) \equiv \int_{a}^{b} p(t$, $\mu) d \mu \leq p<1$

$\left(H_{3}\right) \tau(t, \mu) \in C\left(\left[t_{0}, \infty\right) \times[a, b], R\right)$ is not a decreasing function for $\xi$, and $\tau(t, \mu) \leq t, \lim _{t \rightarrow \infty} \min _{\xi \in[a, b]} \tau(t$, $\mu)=\infty$;

$\left(H_{4}\right) \quad q(t, \xi) \in C\left(\left[t_{0}, \infty\right) \times[c, d],(0, \infty)\right) ;$

$\left(H_{5}\right) g(t, \xi) \in C\left(\left[t_{0}, \infty\right) \times[c, d], R\right)$ is not a decreasing function for $\xi$, such that $g(t, \xi) \leq t$, $\lim _{t \rightarrow \infty} \min _{\xi \in[c, d]} g(t, \xi)=\infty ;$

$\left(H_{6}\right) \quad f(x) \in C(R, R),\left(f(x) / x^{\alpha}\right) \geq \delta>, x \neq 0$.

We also define the following function:

$$
z(t)=x(t)+\int_{a}^{b} p(t, \mu) x[\tau(t, \mu)] d \mu
$$

As far as a solution of (5) is concerned, we mean a nontrivial function $x(t) \in C^{\prime}\left(\left[T_{x}, \infty\right), R\right), T_{x} \geq t_{0}$, which has the property $r(t) z^{\prime \prime}(t) \in C^{\prime}\left(\left[T_{x}, \infty\right)\right)$ and satisfies (5) on $\left[T_{x}, \infty\right)$.

We restrict our attention to those solutions of (5) which satisfy $\sup \{|x(t)|: t \geq T\}>0$ for all $T>T_{x}$. A solution of (5) is said to be oscillatory on $\left[T_{x}, \infty\right)$ if it is neither eventually positive nor eventually negative. Otherwise it is called nonoscillatory.

The rest of this paper is organized as follows. In Section 2, we will present some lemmas which are useful for the proof of our main results. In Section 3, we present new criteria of Philos type for oscillation or certain asymptotic behavior of nonoscillatory solutions of (5).

\section{Several Lemmas}

Lemma 1. Let $x(t)$ be a positive solution of (5), and $r^{\prime}(t) \geq$ $0, z^{\prime \prime \prime}(t)<0$. Then $z(t)$ which is defined as in (6) has only one of the following two properties:

$$
\begin{gathered}
\text { (I) } z(t)>0, z^{\prime}(t)>, z^{\prime \prime}(t)>0 \\
\text { (II) } z(t)>0, z^{\prime}(t)<0, z^{\prime \prime}(t)>0 .
\end{gathered}
$$

Proof. Letting $x(t)$ be a positive solution of (5) on $\left[t_{0}, \infty\right)$, from (6), we have $z(t)>x(t)>0$ and $\left(r(t)\left(z^{\prime \prime}(t)\right)^{\alpha}\right)^{\prime}=$ $-\int_{c}^{d} q(t, \xi) f^{\alpha}(x[g(t, \xi)]) d \xi<0$. Then $r(t)\left(z^{\prime \prime}(t)\right)^{\alpha}$ is a decreasing function and of one sign, and following $\alpha \in(0,1)$ and $\alpha=p / q$ where $p$ and $q$ are odd positive integers, we have that $\left(z^{\prime \prime}(t)\right)^{\alpha}$ and $z^{\prime \prime}(t)$ have the same sign, so $z^{\prime \prime}(t)$ is either eventually positive or eventually negative on $t \geq t_{1} \geq t_{0}$; that is, $z^{\prime \prime}(t)<0$ or $z^{\prime \prime}(t)>0$. If $z^{\prime \prime}(t)<0$, then there exists a constant $M>0$, such that $r(t) z^{\prime \prime} \leq-M<0$. By integrating from $t_{1}$ to $t$, we get

$$
z^{\prime}(t) \leq z^{\prime}\left(t_{1}\right)-M \int_{t_{1}}^{t} \frac{1}{r(s)} d s .
$$

Letting $t \rightarrow \infty$ and using $\left(H_{1}\right)$, we have $z^{\prime}(t) \rightarrow-\infty$. Thus $z^{\prime}(t)<0$ eventually; since $z^{\prime \prime}(t)<0$ and $z^{\prime}(t)<0$, we have $z(t)<0$, which contradicts assumption $z(t)>0$, so $z^{\prime \prime}(t)>$ 0 . Therefore, $z(t)$ has only one of the two properties (I) and (II).

Lemma 2. Let $x(t)$ be a positive solution of (5), and correspondingly $z(t)$ has property (II). Assume that

$$
\int_{t_{0}}^{\infty} \int_{v}^{\infty}\left[\frac{1}{r(u)} \int_{u}^{\infty}\left(\int_{c}^{d} q(s, \xi) d \xi\right) d s\right]^{1 / \alpha} d u d v=\infty .
$$

Then

$$
\lim _{t \rightarrow \infty} x(t)=\lim _{t \rightarrow \infty} z(t)=0 .
$$

Proof. Let $x(t)$ be a positive solution of (5). Since $z(t)$ has property (II), then there exists finite $\operatorname{limit}_{t \rightarrow \infty} z(t)=l$. We assert that $l=0$. Assuming that $l>0$, then we have $l<z(t)<l+\epsilon$, for all $\epsilon>0$. Choosing $\epsilon \in(0, l(1-p) / p)$, we obtain

$$
\begin{aligned}
x(t) & =z(t)-\int_{a}^{b} p(t, \mu) x[\tau(t, \mu)] d \mu \\
& >l-\int_{a}^{b} p(t, \mu) x[\tau(t, \mu)] d \mu \\
& \geq l-p(t) z[\tau(t, a)] \\
& \geq l-p(l+\epsilon) \\
& =k(l+\epsilon) \\
& >k z(t),
\end{aligned}
$$

where $k=(l-p(l+\epsilon)) /(l+\epsilon)>0$. Using $\left(H_{6}\right)$ and $x(t)>k z(t)$, from (5), we find that

$$
\left(r(t)\left(z^{\prime \prime}(t)\right)^{\alpha}\right)^{\prime} \leq-k \delta \int_{c}^{d} q(t, \xi) z^{\alpha}(x[g(t, \xi)]) d \xi .
$$

Note that $z(t)$ has property $(I I)$ and $\left(H_{5}\right)$; we have

$$
\begin{aligned}
\left(r(t)\left(z^{\prime \prime}(t)\right)^{\alpha}\right)^{\prime} & \leq-k \delta z^{\alpha}(x[g(t, d)]) \int_{c}^{d} q(t, \xi) d \xi \\
& =-q_{1}(t) z^{\alpha}\left[g_{1}(t)\right],
\end{aligned}
$$

where $q_{1}(t)=k \delta \int_{c}^{d} q(t, \xi) d \xi, g_{1}(t)=g(t, d)$. Integrating inequality (13) from $t$ to $\infty$, we get

$$
\begin{aligned}
& \left(r(t)\left(z^{\prime \prime}(t)\right)^{\alpha}\right) \geq \int_{t}^{\infty} q_{1}(s) z^{\alpha}\left[g_{1}(s)\right] d s, \\
& z^{\prime \prime}(t) \geq\left[\frac{1}{r(t)} \int_{t}^{\infty} q_{1}(s) z^{\alpha}\left[g_{1}(s)\right] d s\right]^{1 / \alpha} .
\end{aligned}
$$


Using $z\left[g_{1}(t)\right] \geq l$, then we have

$$
z^{\prime \prime}(t) \geq\left[\frac{l^{\alpha}}{r(t)} \int_{t}^{\infty} q_{1}(s) d s\right]^{1 / \alpha} .
$$

Integrating inequality (15) from $t$ to $\infty$, we have

$$
-z^{\prime}(t) \geq l \int_{t}^{\infty}\left[\frac{1}{r(u)}\left(\int_{u}^{\infty} q_{1}(s) d s\right)\right]^{1 / \alpha} d u .
$$

Integrating the last inequality from $t_{1}$ to $\infty$, we obtain

$$
z\left(t_{1}\right) \geq l \int_{t_{1}}^{\infty} \int_{v}^{\infty}\left[\frac{1}{r(u)}\left(\int_{u}^{\infty} q_{1}(s) d s\right)\right]^{1 / \alpha} d u d v ;
$$

we have a contradiction with (8) and so it follows that $\lim _{t \rightarrow \infty} x(t)=0$.

Lemma 3 (see [18]). Let $z(t)>0, z^{\prime}(t)>0, z^{\prime \prime}(t) \leq 0, t>t_{0}$. Then, for each $\beta \in(0,1)$, there exists $T_{\beta} \geq t_{0}$ such that

$$
z(g(t)) \geq \beta \frac{g(t)}{t} \cdot z(t), \quad t \geq T_{\beta} .
$$

Lemma 4 (see [19]). Letting $z(t)>0, z^{\prime}(t)>0, z^{\prime \prime} \geq 0$, $r^{\prime}>0, z^{\prime \prime \prime}(t) \leq 0, t \geq T_{\beta}$, then there exist $\gamma \in(0,1)$ and $T_{\gamma} \geq T_{\beta}$ such that

$$
z(t) \geq \gamma t z^{\prime}(t), \quad t \geq T_{\gamma} .
$$

Lemma 5. For all $\alpha>0$, then for all $A>0, B>0$, one has

$$
B u-A u^{(\alpha+1) / \alpha} \leq \frac{\alpha^{\alpha}}{(\alpha+1)^{\alpha+1}} \cdot \frac{B^{\alpha+1}}{A^{\alpha}} .
$$

Proof. Let $u \geq 0, \alpha>0$. We investigate the maximal value and minimal value of the function $f(u)=B u-A u^{(\alpha+1) / \alpha}$.

At first, for all $A>0, B>0$, the derivative of function $f(u)=B u-A u^{(\alpha+1) / \alpha}$ is $f^{\prime}(u)=B-A((\alpha+1) / \alpha) u^{1 / \alpha}$. It is clear that when $u>(B / A)^{\alpha} \cdot(\alpha /(\alpha+1))^{\alpha}$, we have $f^{\prime}(u)<0$, and when $u<(B / A)^{\alpha} \cdot(\alpha /(\alpha+1))^{\alpha}$, we have $f^{\prime}(u)>0$. Hence the function $f(u)=B u-A u^{(\alpha+1) / \alpha}$ attains its maximum value $\left(\alpha^{\alpha} /(\alpha+1)^{\alpha+1}\right) \cdot\left(B^{\alpha+1} / A^{\alpha}\right)$ at $u=(B / A)^{\alpha} \cdot(\alpha /(\alpha+1))^{\alpha}$. This completes the proof.

\section{Main Result}

Theorem 6. Assume that the condition of Lemma 2 holds, and there exists $\rho \in C^{1}\left(\left[t_{0}, \infty\right),(0, \infty)\right)$, such that $\rho^{\prime}>0$ and

$$
\lim _{t \rightarrow \infty} \int_{T}^{t}\left[Q(s)-\frac{\alpha^{\alpha}}{(\alpha+1)^{\alpha+1}} \cdot \frac{B^{\alpha+1}(s)}{A^{\alpha}(s)}\right] d s=\infty,
$$

where

$$
\begin{gathered}
Q(s)=[\beta \gamma \delta(1-p)]^{\alpha} \cdot \frac{\rho(t)}{t^{\alpha}} g^{2 \alpha}(t, c) \int_{c}^{d} q(t, \xi) d \xi, \\
B(s)=\frac{\alpha}{[\rho(s) r(s)]^{1 / \alpha}}, \\
A(s)=\frac{\rho^{\prime}(s)}{\rho(s)} .
\end{gathered}
$$

Then every solution $x(t)$ of (5) either is oscillatory or converges to zero.

Proof. Assume that (5) has a nonoscillatory solution $x(t)$. Without loss of generality we may assume that $x(t)>0, t \geq$ $t_{1}, x[\tau(t, \mu)]>0,(t, \mu) \in\left[t_{1}, \infty\right) \times[a, b] ; x[g(t, \xi)]>$ $0,(t, \xi) \in\left[t_{1}, \infty\right) \times[c, d]$, and $z(t)$ is defined as in (6). By Lemma 1, we have that $z(t)$ has property (I) or property (II). At first, when $z(t)$ has property (I), we obtain

$$
\begin{aligned}
x(t) & =z(t)-\int_{a}^{b} p(t, \mu) x[\tau(t, \mu)] d \mu \\
& \geq z(t)-\int_{a}^{b} p(t, \mu) z[\tau(t, \mu)] d \mu \\
& \geq z(t)-z[\tau(t, b)] \int_{a}^{b} p(t, \mu) d \mu \\
& \geq\left(1-\int_{a}^{b} p(t, \mu) d \mu\right) z(t) \\
& \geq(1-p) z(t) .
\end{aligned}
$$

Using $\left(H_{5}\right)$ and $\left(H_{6}\right)$, we get

$$
\begin{aligned}
(r(t) & \left.\left(z^{\prime \prime}(t)\right)^{\alpha}\right)^{\prime} \\
& \leq-\delta^{\alpha}(1-p)^{\alpha} \int_{c}^{d} q(t, \xi) z^{\alpha}[g(t, \xi)] d \xi \\
& \leq-\delta^{\alpha}(1-p)^{\alpha} z^{\alpha}[g(t, c)] \int_{c}^{d} q(t, \xi) d \xi \\
& \equiv-q_{2}(t) z^{\alpha}\left[g_{2}(t)\right],
\end{aligned}
$$

where

$$
q_{2}(t)=\delta^{\alpha}(1-p)^{\alpha} \int_{c}^{d} q(t, \xi) d \xi, \quad g_{2}(t)=g(t, c) .
$$

Let

$$
w(t)=\rho(t) r(t)\left(\frac{z^{\prime \prime}(t)}{z^{\prime}(t)}\right)^{\alpha}, \quad t \geq t_{1}
$$

Then

$$
\frac{w(t)\left(z^{\prime}(t)\right)^{\alpha}}{\rho(t)}=r(t)\left(z^{\prime \prime}(t)\right)^{\alpha}
$$

so

$$
\begin{gathered}
\left(\frac{w(t)\left(z^{\prime}(t)\right)^{\alpha}}{\rho(t)}\right)^{\prime}=\left(r(t)\left(z^{\prime \prime}(t)\right)^{\alpha}\right)^{\prime} \leq-q_{2}(t) z^{\alpha}\left[g_{2}(t)\right], \\
w^{\prime}(t) \leq-q_{2}(t) \rho(t)\left[\frac{z\left[g_{2}(t)\right]}{z^{\prime}}\right]^{\alpha} \\
+\frac{\rho^{\prime}(t)}{\rho(t)} w(t)-\frac{\alpha}{[\rho(t) r(t)]^{1 / \alpha}} w^{(\alpha+1) / \alpha}(t) .
\end{gathered}
$$


Letting $u(t)=z^{\prime}(t)$, from Lemma 3, we obtain

$$
\frac{1}{z^{\prime}(t)} \geq \frac{\beta g_{2}(t)}{t z^{\prime}\left[g_{2}(t)\right]}, \quad t \geq T_{\beta} \geq t_{1} \text {. }
$$

Using Lemma 4, we get

$$
z\left[g_{2}(t)\right] \geq \gamma g_{2}(t) z^{\prime}\left[g_{2}(t)\right], \quad t \geq T_{\gamma} \geq T_{\beta} .
$$

Hence

$$
\begin{array}{r}
w^{\prime}(t) \leq-Q(t)+\frac{\rho^{\prime}(t)}{\rho(t)} w(t)-\frac{\alpha}{[\rho(t) r(t)]^{1 / \alpha}} w^{(\alpha+1) / \alpha}(t), \\
t \geq T_{\gamma},
\end{array}
$$

where $Q(t)$ is defined as (21). Letting $A(t)=\rho^{\prime}(t) / \rho(t), B(t)=$ $\alpha /(\rho(t) r(t))^{1 / \alpha}$, we have that

$$
w^{\prime}(t) \leq-Q(t)+A(t) w(t)-B(t) w^{(\alpha+1) / \alpha}(t),
$$

and, from Lemma 5, we obtain

$$
w^{\prime}(t) \leq-Q(t)+\frac{\alpha^{\alpha}}{(\alpha+1)^{\alpha+1}} \cdot \frac{B^{\alpha+1}(t)}{A^{\alpha}(t)} .
$$

Integrating inequality (33) from $T$ to $t$,

$$
\int_{T}^{t} w^{\prime}(s) d s \leq-\int_{T}^{t}\left(Q(s)-\frac{\alpha^{\alpha}}{(\alpha+1)^{\alpha+1}} \cdot \frac{B^{\alpha+1}(s)}{A^{\alpha}(s)}\right) d s,
$$

we obtain

$$
0<w(t) \leq w(T)-\int_{T}^{t}\left(Q(s)-\frac{\alpha^{\alpha}}{(\alpha+1)^{\alpha+1}} \cdot \frac{B^{\alpha+1}(s)}{A^{\alpha}(s)}\right) d s,
$$

which contradicts (21). If $z(t)$ has property (II), since (8) holds, then the conditions in Lemma 2 are satisfied. Hence $\lim _{t \rightarrow \infty} x(t)=0$.

This completes the proof.

\section{Conflict of Interests}

The authors declare that there is no conflict of interests regarding the publication of this paper.

\section{Acknowledgments}

This work is supported by the Scientific Research Funds of Hunan Provincial Science and Technology Department of China (no. 12FJ4252 and no. 2013SK3143), National Natural Science Foundation of China (nos. 11101053, 11326116).

\section{References}

[1] C. Huang, Z. Yang, T. Yi, and X. Zou, "On the basins of attraction for a class of delay differential equations with nonmonotone bistable nonlinearities," Journal of Differential Equations, vol. 256, no. 7, pp. 2101-2114, 2014.
[2] C. Huang, C. Peng, X. Chen, and F. Wen, "Dynamics analysis of a class of delayed economic model," Abstract and Applied Analysis, vol. 2013, Article ID 962738, 12 pages, 2013.

[3] G. Qin, C. Huang, Y. Xie, and F. Wen, "Asymptotic behavior for third-order quasi-linear differential equations," Advances in Difference Equations, vol. 2013, article 305, 2013.

[4] C. Huang, H. Kuang, X. Chen, and F. Wen, "An LMI approach for dynamics of switched cellular neural networks with mixed delays," Abstract and Applied Analysis, vol. 2013, Article ID 870486, 8 pages, 2013.

[5] R. P. Agarwal, S. Grace R, and D. O'Regan, Oscillation Theory for Difference and Functional Differential Equations, Kluwer Academic, Dordrecht, The Netherlands, 2000.

[6] A. Tiryaki and M. F. Aktaş, "Oscillation criteria of a certain class of third order nonlinear delay differential equations with damping," Journal of Mathematical Analysis and Applications, vol. 325, no. 1, pp. 54-68, 2007.

[7] R. P. Agarwal, S. R. Grace, and D. O'Regan, Oscillation Theory for Second Order Dynamic Equations, vol. 5, Taylor and Francis, London, UK, 2003.

[8] L. H. Erbe, Q. Kong, and B. G. Zhang, Oscillation Theory for Functional-Differential Equations, Marcel Dekker, New York, NY, USA, 1995.

[9] P. G. Wang, "Oscillation criteria for second-order neutral equations with distributed deviating arguments," Computers \& Mathematics with Applications, vol. 47, no. 12, pp. 1935-1946, 2004.

[10] Z. L. Han, S. R. Sun, and B. Shi, "Oscillation criteria for a class of second-order Emden-Fowler delay dynamic equations on time scales," Journal of Mathematical Analysis and Applications, vol. 334, no. 2, pp. 847-858, 2007.

[11] M. F. Aktas, A. Tiryaki, and A. Zafer, "Oscillation criteria for third-order nonlinear functional differential equations," Applied Mathematics Letters, vol. 23, no. 7, pp. 756-762, 2010.

[12] S. R. Grace, R. P. Agarwal, R. Pavani, and E. Thandapani, "On the oscillation of certain third order nonlinear functional differential equations," Applied Mathematics and Computation, vol. 202, no. 1, pp. 102-112, 2008.

[13] S. R. Grace, R. P. Agarwal, and M. F. Aktas, "On the oscillation of third order functional differential equations," Indian Journal of Pure and Applied Mathematics, vol. 39, no. 6, pp. 491-507, 2008.

[14] N. Parhi and S. Padhi, "Asymptotic behaviour of solutions of third order delay-differential equations," Indian Journal of Pure and Applied Mathematics, vol. 33, no. 10, pp. 1609-1620, 2002.

[15] A. Tiryaki and S. Yaman, "Asymptotic behaviour of a class of nonlinear functional differential equations of third order," Applied Mathematics Letters, vol. 14, no. 3, pp. 327-332, 2001.

[16] Y. Qiu, "Philos Oscillation theorems for third-order neutral equations," Mathematics in Practice and Theory, vol. 41, pp. 247252, 2011.

[17] Q. Zhang, L. Gao, and Y. Yu, "Oscillation criteria for third-order neutral differential equations with continuously distributed delay," Applied Mathematics Letters, vol. 25, no. 10, pp. 1514-1519, 2012.

[18] L. Erbe, "Oscillation criteria for second order nonlinear delay equations," Canadian Mathematical Bulletin, vol. 16, pp. 49-56, 1973.

[19] I. T. Kiguradze, "On the oscillation of solution of the equation $d^{m} u / d t^{m}+a(t)|u|^{m}$ signu $=0$," Matematicheskii Sbornik, vol. 65, pp. 172-187, 1964. 


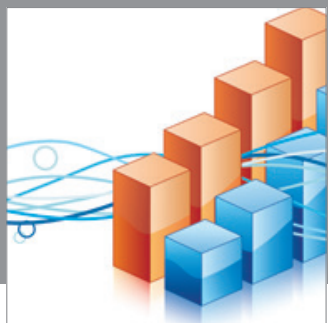

Advances in

Operations Research

mansans

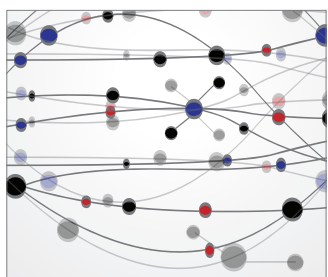

The Scientific World Journal
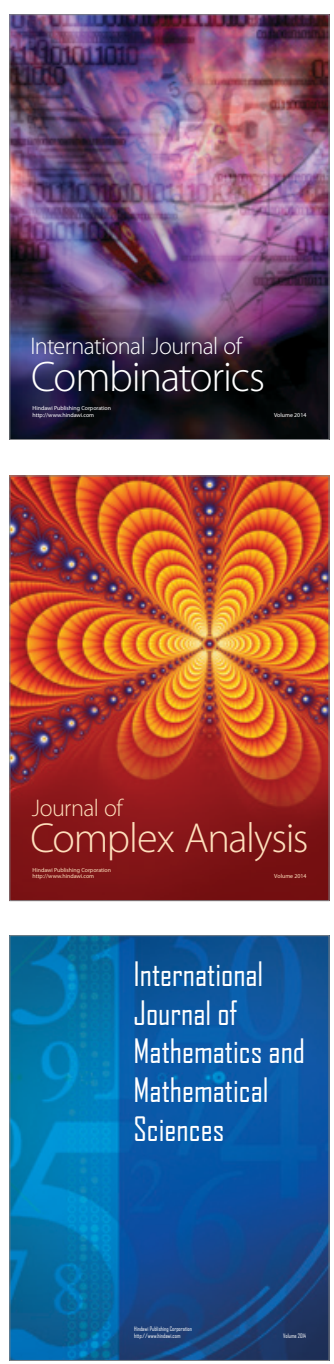
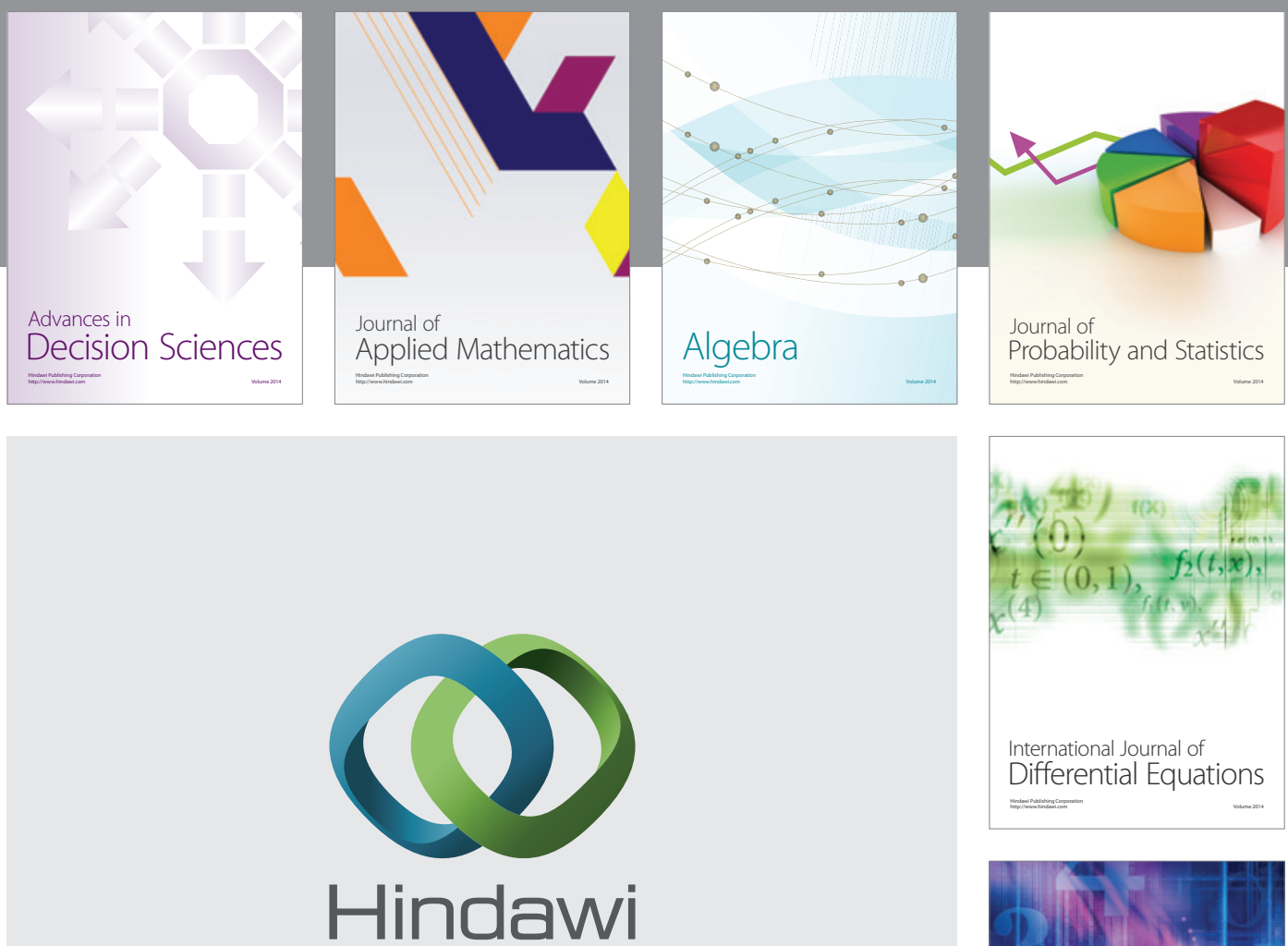

Submit your manuscripts at http://www.hindawi.com
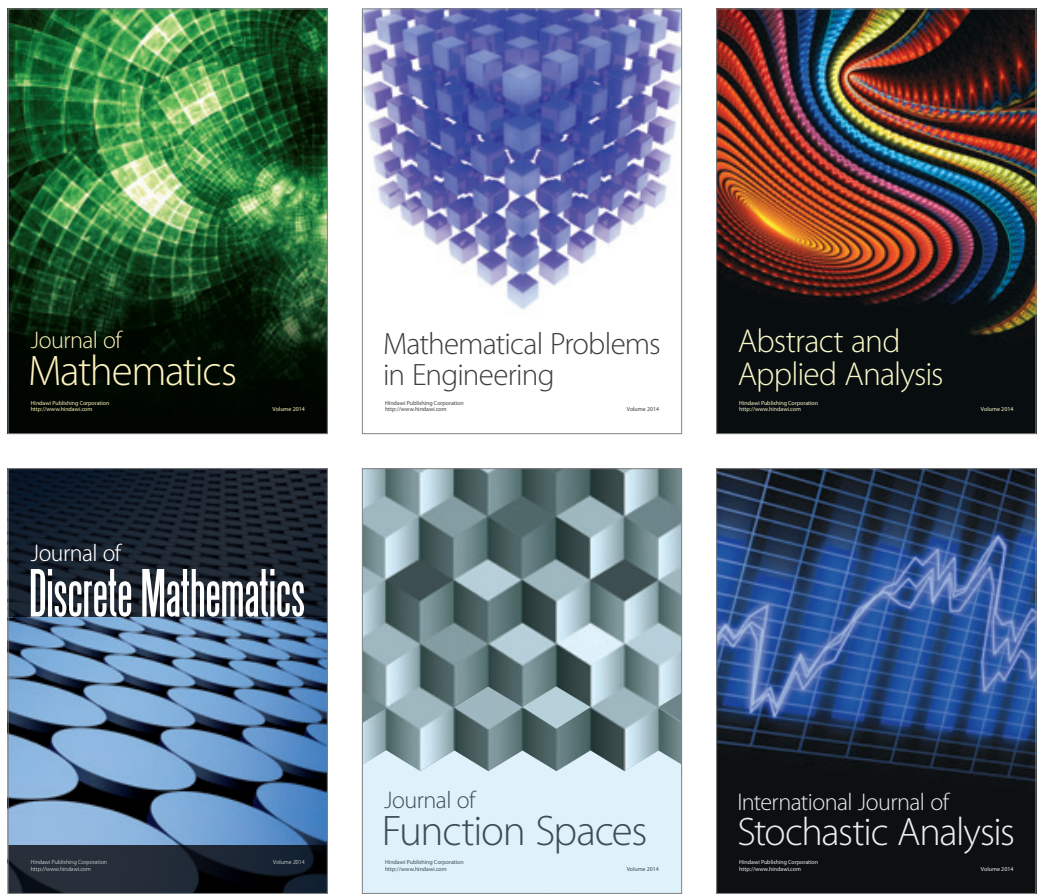

Journal of

Function Spaces

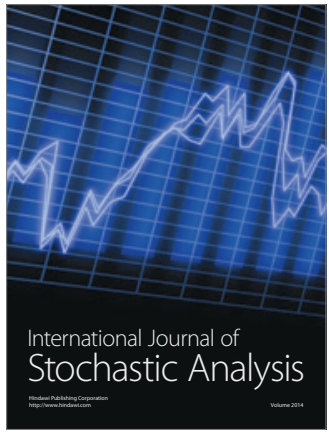

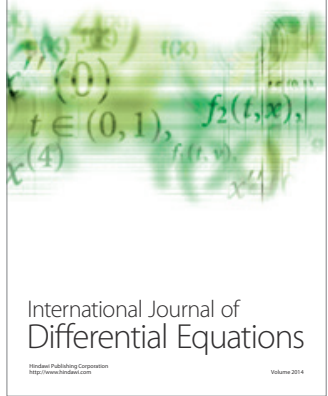
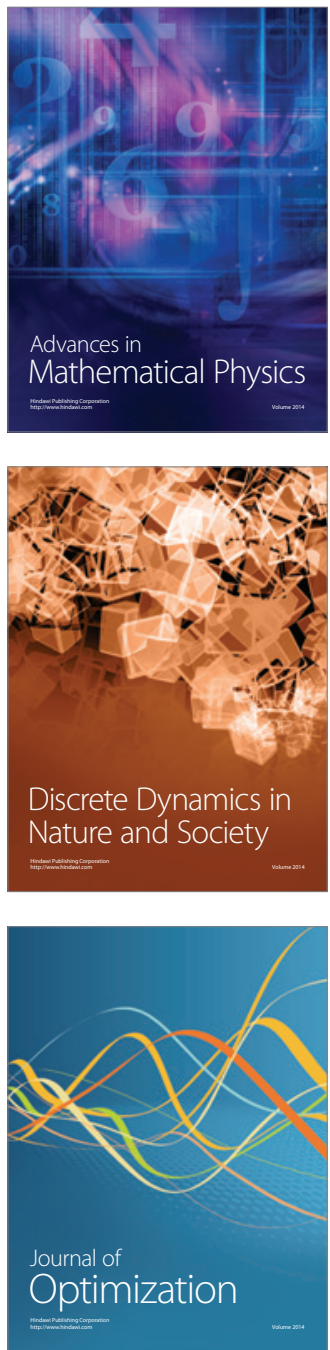